\title{
Digues en Caissons Perforés: du Front de Mer de Beyrouth au nouveau port de Tanger
}

\author{
Pierre Aristaghes ${ }^{(a)}$, Valérie Blanchet ${ }^{(\mathrm{b})}$, Nicolas Berthe ${ }^{(\mathrm{c}),}$ Bouygues $\mathrm{TP}^{(\mathrm{d})}$
}

\author{
(a)Directeur Etudes et Développements 0130604604 \\ p.aristaghes@bouyques-construction.com \\ (b)Chef de Service Adjoint Etudes Ports et Environnement 0130605288 \\ v.blanchet@bouygues-construction.com \\ (c)Ingénieur d'Etudes 0130605784 \\ n.berthe@bouyques-construction.com \\ (d)Challenger - 78 - St Quentin en Yvelines cedex
}

\section{Résumé:}

L'article décrit deux projets en conception-construction qui ont en commun de mêler digues à talus et caissons perforés, ceux-ci répondant à des contraintes spécifiques : une promenade à plusieurs niveaux jouant également le rôle de barrière anti-franchissements pour le front de mer de Beyrouth, une digue portuaire en eau profonde pour le nouveau port de Tanger Méditerranée.

\section{Abstract:}

The paper describes two design and build projects, which both combine rubble mound breakwaters with perforated caissons. These caissons have specific functions : a pedestrian multilevel walkway also acting as an anti-overtopping structure for the Beirut Sea Front, a deep water breakwater for the new port of Tangiers on Mediterranean Sea.

Mots-clés: Digue, Houle, Caisson, Stabilité, Franchissement

\section{Introduction}

Les digues en caissons présentent des caractéristiques très différentes des digues à talus (ref 1), et le choix entre l'une ou l'autre structure dépend de nombreux facteurs parmi lesquels les conditions de site (profondeur, terrain de fondation, encombrement), les fonctionnalités attendues, les coûts locaux (main d'œuvre, matériaux, matériels) et l'environnement jouent un rôle majeur.

Le présent article présente deux projets majeurs, réalisés en conception-construction sur la base de variantes larges, où les caissons ont apporté des solutions élégantes à des problématiques très spécifiques.

Le premier projet est le Front de Mer de Beyrouth, achevé en 1997, qui s’inscrit dans la reconstruction du Centre Ville; le second est la digue du nouveau port de Tanger Méditerranée, en cours de réalisation.

\section{Front de Mer de Beyrouth}

\subsection{Contexte}

Après plusieurs années de guerre, une ancienne baie naturellement protégée des houles s'est retrouvée comblée par des décharges de détritus divers, offrant de fait un nouveau trait de côte exposé aux houles du large. 
Afin de permettre la reconstruction d'un nouveau centre de prestige, un appel d'offres en conception-construction fut lancé pour un ouvrage offrant les fonctions suivantes :

- front de mer piétonnier sur un peu plus d'un kilomètre

- barrière anti-franchissement (efficace à $100 \%$ pour $\mathrm{Hs}=7.4 \mathrm{~m}$ et $\mathrm{Tp}=15 \mathrm{~s}$, limitant les débits à $30 \mathrm{l} / \mathrm{m} / \mathrm{s}$ pour $\mathrm{Hs}=9 \mathrm{~m}$ )

- niveau maximum de $5.5 \mathrm{~m}$ au-dessus des plus hautes eaux

- structures invisibles sauf promenades

Le design de référence consistait en une double barrière, constituée d'une première ligne de caissons arasés à $50 \mathrm{~cm}$ sous le niveau de la mer, placé à une cinquantaine de mètres d'une seconde ligne de caissons adossée au terre-plein. L'un des points faibles de ce design, qui se révéla tardivement à l'issue de la campagne géotechnique en mer, était son extrême sensibilité aux tassements.

\subsection{Conception générale de la solution variante}

L’idée de la variante consistait :

- à remplacer la première ligne de caissons en mer par un platier sous-marin, suffisamment large par rapport à la longueur d'onde des houles pour les briser efficacement avant qu'elles n'atteignent la seconde ligne de caissons ;

- à concevoir ces caissons-promenade comme des «trappes à vagues », afin de réduire le franchissement des vagues résiduelles en bout de platier.

Ce concept est représenté ci-dessous, le platier, d'une largeur d'une centaine de mètres, étant arasé aux environs de $-5 \mathrm{CM}$ :

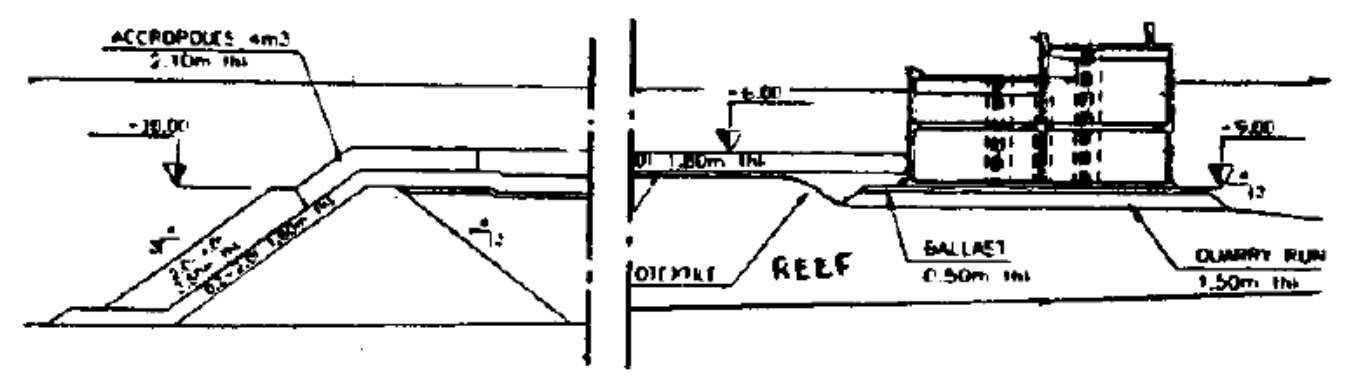

Ce platier fut prédimensionné lors de la préparation de l’offre sur la base d'essais en canal à houle chez Oceanide, y compris le design global des 80 caissons, tel que représenté cidessous, où l'on distingue :

- une promenade à niveau bas (1.5 CM), avec un « banc chasse-mer » ;

- une promenade à +4.5 CM au-dessus d'une chambre de dissipation ;

- deux séries de fentes (porosités 50 puis 30\%) fermant cette chambre

- un parapet supérieur à la cote maximale autorisée (+6.5 CM)

- un radier fondé à -12 CM, soit une hauteur totale de plus de 18 m. 


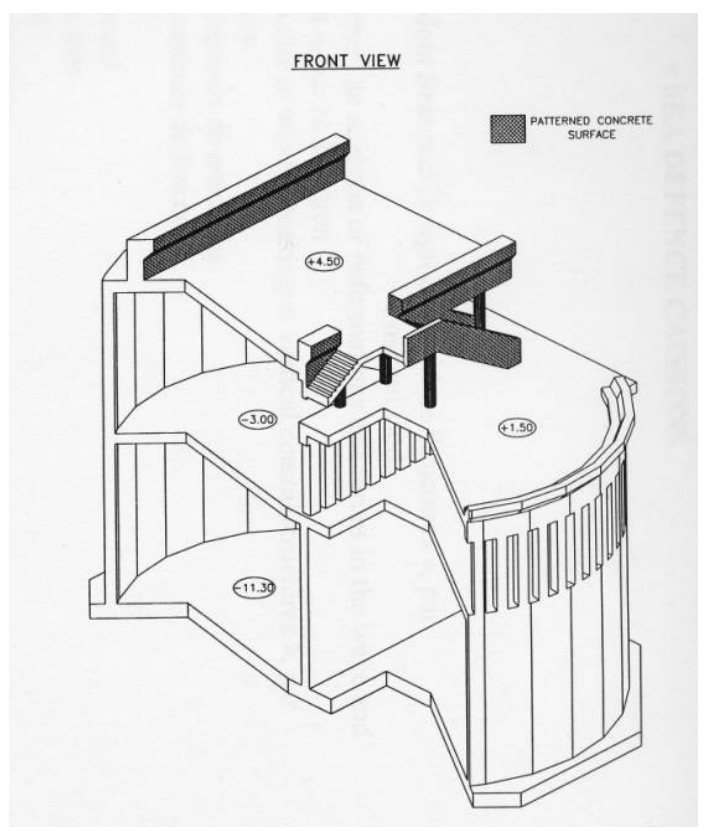

Les essais en canal effectués à cette phase visaient exclusivement à valider la faisabilité du concept vis-à-vis des franchissements, et à optimiser le caisson de ce point de vue : c'est à ce stade qu'ont été imaginés le « banc chasse-mer » et la suppression totale de la paroi entre les deux dalles-promenade, qui, à eux deux, ont augmenté de $40 \%$ les hauteurs de houle de début de franchissement pour une géométrie de platier donnée.

\subsection{Spécificités des études de détail}

Cette structure diffère des digues verticales classiques par les points suivants :

- caisson partiellement enterré dans un platier (de -12 à - $5 \mathrm{CM}$ ), rendant inapplicables les formules classiques donnant les efforts de houle, du fait d'une cinématique de houle fortement perturbée par le platier (de fait, les essais préliminaires ont montré que la largeur du platier devait être de l'ordre de $100 \mathrm{~m}$ pour que les vagues soient brisées sans provoquer de déferlement plongeant au droit du caisson mais systématiquement devant ; pour des houles de $15 \mathrm{~s}$ de période et des fonds devant le platier de 20 à $25 \mathrm{~m}$, donc des longueurs d'onde de l'ordre de $200 \mathrm{~m}$, cette conclusion équivaut à imposer une largeur de platier de l'ordre d'une demi-longueur d'onde, ce qui est a posteriori logique) ;

- ouvrage "urbain », supposé ne subir que des déplacements faibles, nécessitant une approche plus poussée que les simples vérifications de sécurité au glissement et au renversement auxquelles on se limite pour des digues classiques, pour lesquelles on admet généralement des tassements absolus et différentiels importants ;

- ouverture totale aux vagues (pour des raisons de réduction des franchissements, la chambre arrière jouant le rôle de trappe à vagues), induisant des sous-pressions sous les dalles-promenade, nécessitant une approche fine des pressions hydrodynamiques à différentes phases de l'attaque par les vagues.

L’étude structurelle du caisson a nécessité une approche expérimentale en canal (à Delft), où l'on a non seulement mesuré les efforts globaux via une balance dynamométrique, mais aussi les pressions à $200 \mathrm{~Hz}$ en une vingtaine de points représentés ci-dessous : 


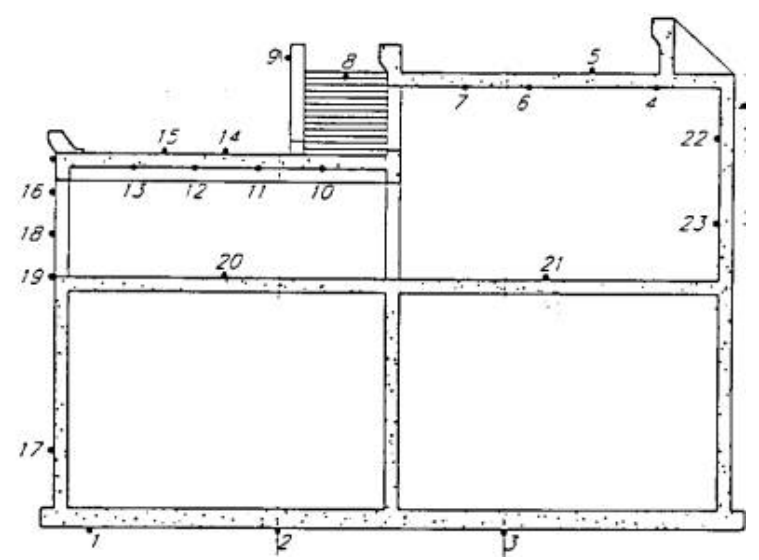

De fait, lors des essais préliminaires effectués chez Oceanide, on avait procédé à des mesures à $1000 \mathrm{~Hz}$ avec deux capteurs de pression dans les chambres (parois verticales intérieures et sous-faces des dalles) que l'on déplaçait d'un essai à l'autre, dans l'objectif de distinguer les phénomènes de type ondes de gravité (se propageant avec une célérité de l'ordre de celle des vagues, soit entre ) des phénomènes de choc avec occlusion d'air (célérité, calculée sur la base des déphasages entre les pics mesurés par les deux capteurs, très supérieure à celle-ci car plus liée à la célérité du son).

Dans la première chambre, on avait relevé des phénomènes du premier type lors de la progression de l'onde des points 13 vers 10 (cf schéma précédent), et du second type en fond de chambre, du fait du blocage momentané de la vague par la retombée de poutre coiffant le second voile perforé (se traduisant par un décalage systématique des pics de moins de $0.002 \mathrm{~s}$ entre deux points distants de $12 \mathrm{~cm}$ en modèle, soir une célérité équivalente de plus de $60 \mathrm{~m} / \mathrm{s}$ en modèle, à comparer à la célérité des ondes de gravité en modèle, à savoir (gh) ${ }^{1 / 2}<2 \mathrm{~m} / \mathrm{s}$ pour $40 \mathrm{~cm}$ d'eau en pied de platier).

Dans la seconde chambre, on observe deux types de phénomènes : dans un premier temps, pour les vagues les plus fortes, des effets d'impact non concomitants (de type " hammer shock ») du fait de l'entrée directe de vagues par la "trappe » située entre les deux promenades; dans un second temps, un remplissage progressif de la chambre par la paroi perforée à $30 \%$ séparant les deux chambres, se terminant de temps en temps par un « compression shock » lorsque ce remplissage va jusqu’à piéger de l'air en partie supérieure du fond de chambre.

On a aussi profité de ces essais préliminaires à $1000 \mathrm{~Hz}$ pour évaluer la fréquence minimale d'échantillonnage, à savoir celle qui permet de ne pas manquer un pic d'une part, et qui permet de distinguer les deux types de phénomènes d'autre part. Il s'est avéré que l'on commençait à dégrader l'information à partir de $100 \mathrm{~Hz}$. D'où le choix de travailler à $200 \mathrm{~Hz}$ sur la vingtaine de capteurs de Delft.

Ces mesures et des vidéos ont permis de confirmer la chronologie des événements liés à une vague, et de mettre en évidence la concomitance des pics de pression, et les phénomènes d'occlusion d'air, caractérisés par une simultanéité des pics en des points situés dans une même chambre, qui ont fait l'objet d'une correction d'échelle par rapport à la similitude de Froude, en adaptant la formulation de Lundgren (ref 2), à savoir :

Un volume V d'eau impactant avec une vitesse u un volume Ao d'air, initialement à la pression atmosphérique Po, va, du fait que cet air est piégé, le comprimer jusqu’à un volume final $A_{\min }$, tel que l'énergie de compression équilibre l'énergie cinétique de la masse d'eau :

$$
-\int_{A o}^{A_{\min }}\left(P-P_{o}\right) \cdot d A=E_{c o}=1 / 2 \rho V u^{2}
$$


Etant donné la brièveté du phénomène, on peut supposer la compression adiabatique $\left(\mathrm{Pa}^{\gamma}\right.$ $=$ cte), ce qui conduit à l'expression suivante où $\mathrm{P}_{\max }$ désigne la pression de pic en fin de compression :

$$
f\left(P_{o}, P_{\max }\right)=\int_{P_{o}}^{P_{\max }}\left(P-P_{o}\right) \cdot P^{-1 / \gamma-1} d P=\frac{\gamma \rho V u^{2}}{2 A_{o} P_{o}^{1 / \gamma}}
$$

Compte-tenu de ce que la pression atmosphérique est identique en modèle et en prototype, il en résulte, entre la pression Pmax réelle et la pression maximale mesurée en modèle Pmod, une relation du type suivant, $\lambda$ désignant l'échelle du modèle (de l'ordre de 50 dans notre cas) :

$$
\mathrm{f}\left(\mathrm{Po}, \mathrm{P}_{\mathrm{max}}\right)=\lambda . \mathrm{f}\left(\mathrm{Po}, \mathrm{P}_{\mathrm{mod}}\right)
$$

d'où l'on déduit une correction sur les suppressions, fonction de l'échelle mais aussi de la valeur des surpressions, qui se traduit dans notre cas par une réduction d'un facteur d'au moins 5 du pic par rapport à la stricte application de la similitude de Froude, soit :

$$
\left(\mathrm{P}_{\text {max }}-\mathrm{Po}\right) \approx \lambda .\left(\mathrm{P}_{\bmod }-\mathrm{Po}\right) / 5
$$

Cette correction a été appliquée sur la partie au-delà des niveaux « quasistatiques », pour les pics identifiés comme relevant d’un « compression shock », comme illustré ci-dessous :

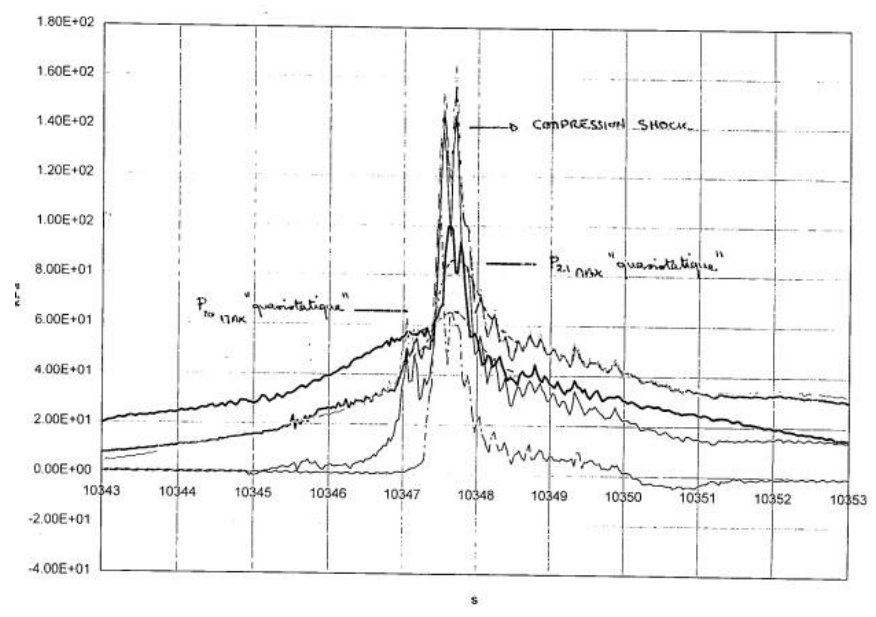

Enfin, les franchissements et la stabilité du platier, protégé par des accropodes et des enrochements, ainsi que celle de la digue en accropodes protégeant une marina située en extrémité du front de mer, ont été testés en cuve à houle à Delft : 


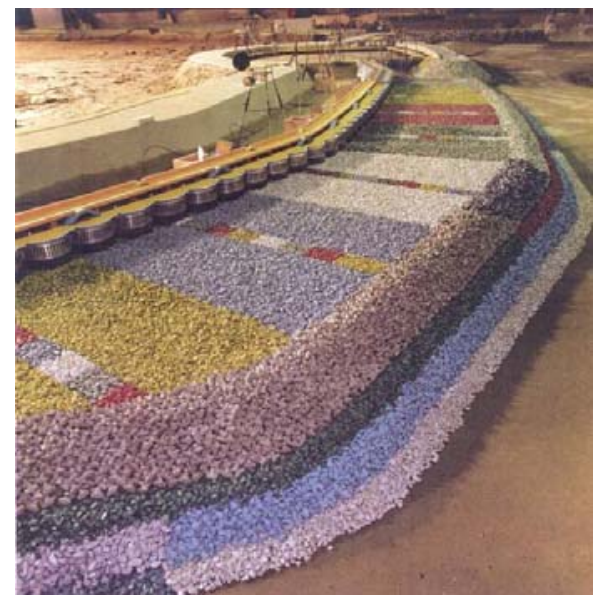

Entre autres problèmes rencontrés à ce stade, on peut mentionner :

- la difficulté de reconstituer une houle incidente réaliste sur l'ensemble du modèle (qui occupait une bonne vingtaine de mètres de la marina à l'extrémité du platier), du fait de la nécessité de tronquer la bathymétrie à une profondeur (de l'ordre de $40 \mathrm{~m}$ ) où la réfraction a déjà joué en nature ;

- la difficulté de définir de manière objective le «début de franchissement » en cuve à houle, ce qui a amené à développer une grille détectrice, constituée de fils parallèles ne déclenchant un signal qu'en cas de mise en contact par de l'eau verte.

\subsection{Aspects architecturaux}

Compte-tenu de son insertion dans la ville, l’ouvrage a, dès la phase de préparation de l'offre, d'une concertation étroite entre ingénieurs hydrauliciens et structures d'une part, urbanistes et architectes d'autre part, comme l'illustre l'image suivante (architecte Sato) réalisée pour la présentation de l’offre :

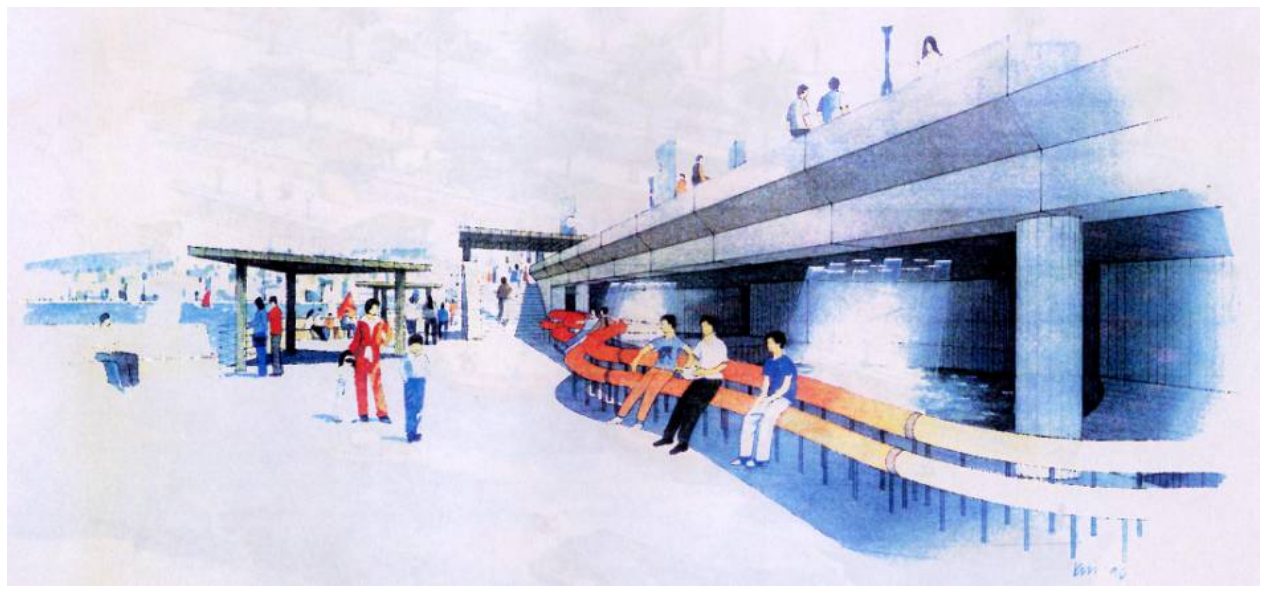

que l'on peut comparer avec les vues suivantes, prises respectivement avant finition du platier puis une fois l'ouvrage terminé : 


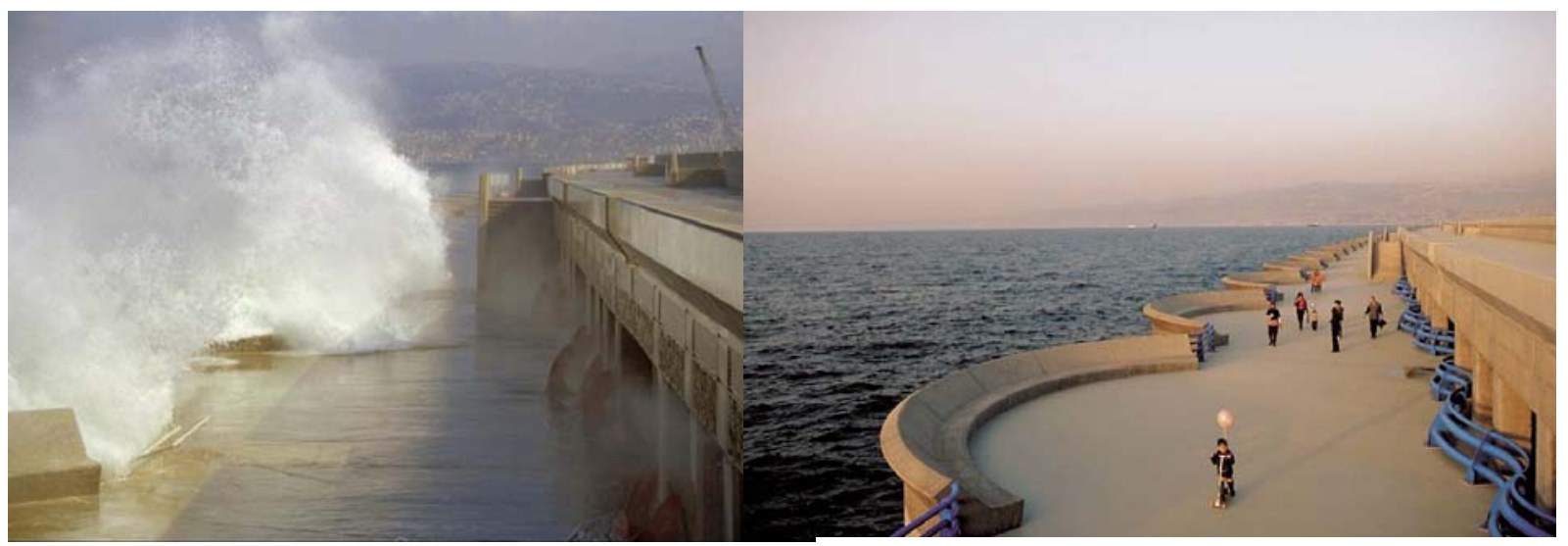

\section{Nouveau port de Tanger Méditerranée}

\subsection{Contexte}

Dans le cadre d'un projet de création d'un nouveau port à l'est de Tanger, principalement dédié au trafic conteneurs, l’Agence Spéciale Tanger Méditerranée (ASTM) a lancé un appel d'offres pour la construction des ouvrages de protection.

La digue principale, longue de plus de $2 \mathrm{~km}$, est censée résister à des houles centennales allant jusqu'à $9 \mathrm{~m}$ de hauteur significative pour des périodes relativement longues, allant jusqu'à 18s.

Elle était prévue en tétrapodes, avec un musoir par des fonds de plus de $40 \mathrm{~m}$, protégé par des blocs de $20 \mathrm{~m} 3$ en béton alourdi disposés selon une pente de $2 / 1$.

La variante retenue a consisté en un remplacement des tétrapodes par des accropodes (jusqu'à $16 \mathrm{~m}^{3}$ ) entre l'enracinement et les fonds d'environ-20 CM, et par des caissons audelà (cf schéma suivant, où les caissons sont en rouge) :

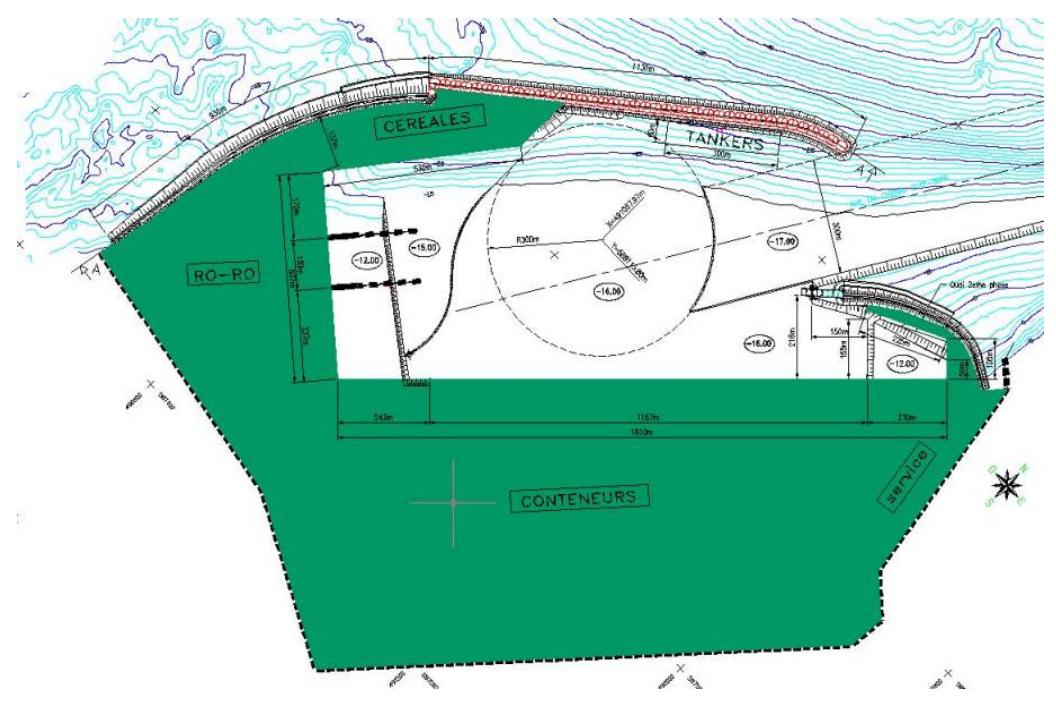




\subsection{Conception des caissons}

Sur la base de prédimensionnements manuels et de considérations liées aux méthodes de construction et de mise à l'eau, la structure des caissons a été définie comme représenté sur la vue suivante.

L'embase des caissons (de -20 à +4 CM) est constituée d'une structure quadrilobée, destinée à être remblayée une fois en place.

Sur cette embase sont ensuite coulées :

- une dalle à + 4.5 CM côté port,

- une chambre cylindrique jusqu'à +12 CM, perforée côté mer

- un mur chasse-mer arasé à +15 CM au sommet côté arrière.

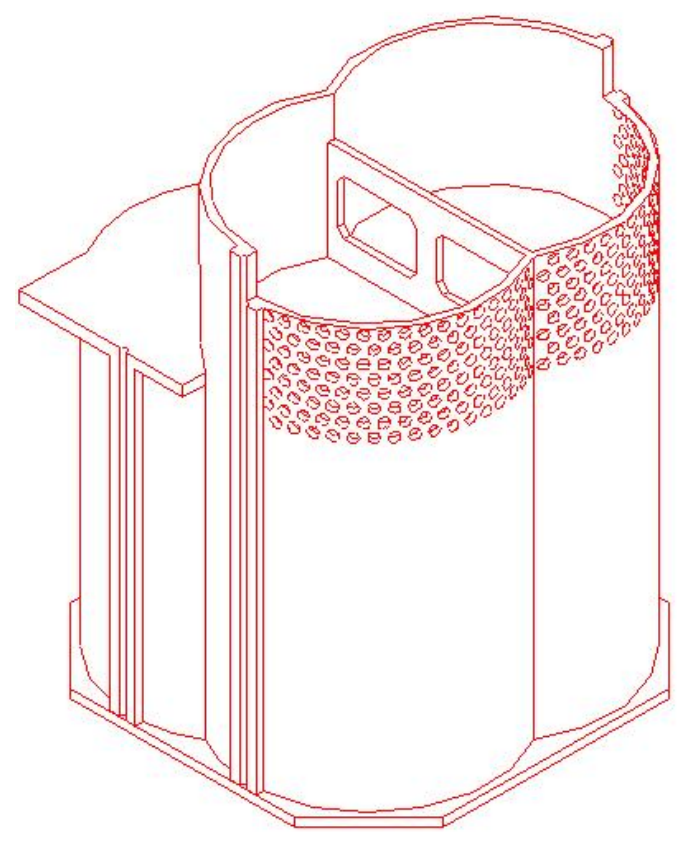

\subsection{Impact des caissons sur le plan-masse}

En termes de plan-masse, les caissons offraient un avantage important par rapport aux digues à talus.

En effet, notamment au droit du musoir, la profondeur était telle que l'emprise au sol de la digue à talus était très importante, comme illustré par la superposition suivante en section courante, qui illustre l'impact environnemental de la variante (réduction d'un facteur 4 des matériaux de carrière et des emprises au sol) :

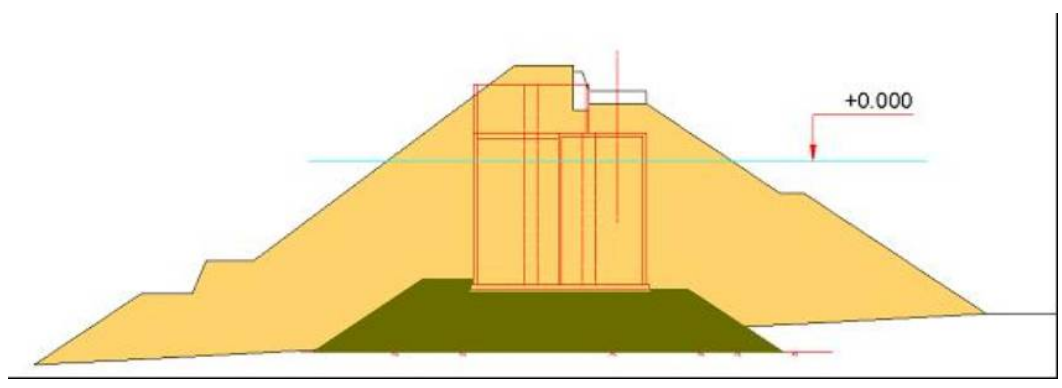


Par suite, le chenal utile (c'est-à-dire la partie à -17 CM) se trouvait très éloignée de l'axe de la digue, d'où, à implantation identique, une moins bonne protection du plan d'eau qu'avec les caissons, la passe ouverte à la pénétration des houles étant nettement plus large que la passe utile.

De plus, le caisson est plus flexible en termes d'adaptation à une fonction donnée, notamment au droit du terre-plein céréalier, qui se trouve en continuité avec la dalle de roulement.

\subsection{Stabilité et aspects structurels}

Cette structure a été testée en canal chez Oceanide durant la préparation de l'offre, afin d'évaluer les efforts globaux, d'une manière similaire à ce qui a été décrit plus haut pour le front de Mer de Beyrouth. Les franchissements ont également été mesurés, et ont prouvé le meilleur comportement, à cote d'arase égale, des caissons par rapport à la digue à talus (rapport entre 10 et 100 sur les débits).

Le graphique suivant donne les franchissements mesurés pour plusieurs hauteurs significatives (et pour des périodes de pic de $18 \mathrm{~s}$ et un niveau de mer à $+2.3 \mathrm{~m} \mathrm{CM}$ ), et ce pour 3 configurations du caisson, dont la configuration retenue (triangles rouges). Ceci permet d'apprécier l'effet :

- des perforations : la courbe rose correspond à un caisson dont les perforations ne règnent qu'au-dessus de la cote $+6.38 \mathrm{CM}$ (soit une face imperméable se prolongeant d'environ $2 \mathrm{~m}$ plus haut que le caisson de référence); on observe une majoration d'un facteur 2 à 3 des franchissements ;

- du mur chasse-mer entre les niveaux +12 et +15 m CM, qui réduit d'un facteur 10 les franchissements.

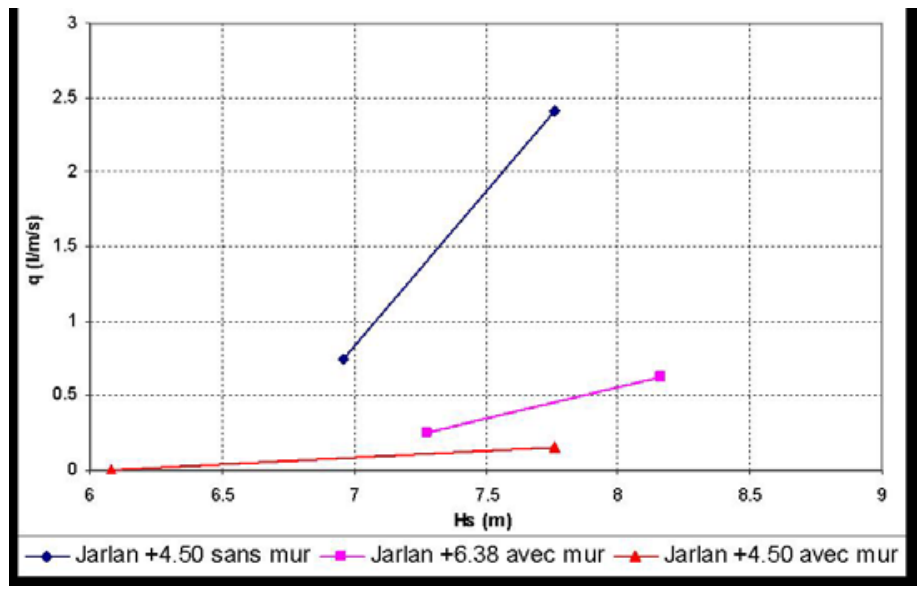

L'analyse fine de certaines vagues critiques (vidéos et capteurs de pressions) a permis de valider la description des phénomènes, plus simple que celle du projet précédent, et donc les répartitions de pressions utilisées pour le calcul structurel.

La connexion avec la digue à talus a fait l'objet d'une attention particulière, l'idée étant de buter la carapace en accropodes par le premier caisson, complété par un lobe additionnel destiné à buter latéralement la partie supérieure de la digue à talus. Cette connexion, qui a été testée en cuve à houle chez Sogreah (cf photo ci-dessous), n’a posé aucun problème ni de stabilité ni de concentration de franchissements, les houles n'attaquant jamais dans le sens caisson vers talus. 


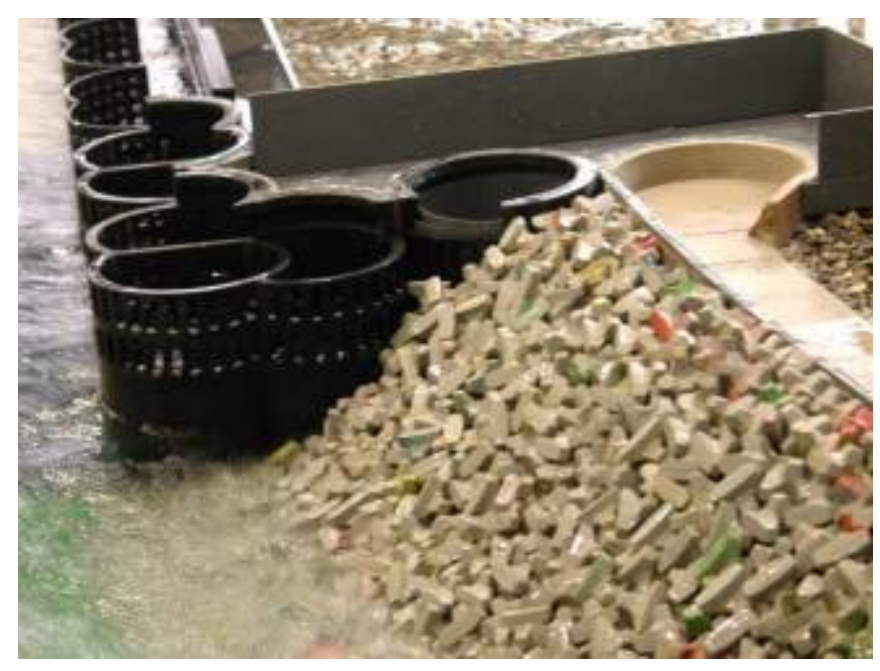

On voit sur cette photo que les trous circulaires des caissons ont été remplacés par des fentes verticales. A porosité égale, le comportement est très similaire, le seul effet notable étant un début de remplissage (stabilisateur) très légèrement plus rapide avec les fentes. De fait, comme sur le front de mer de Beyrouth, les fentes permettent de simplifier le ferraillage et d'améliorer, notamment dans le cas d'un coffrage glissant, la qualité du bétonnage.

\section{Conclusion}

Les deux projets précédents constituent deux références importantes, qui illustrent les atouts des solutions caissons et leur complémentarité avec les digues à talus.

\section{Références:}

1.Aristaghes P., Bouttes, J.M.Lannuzel : les digues en caisson perforés (Bull AIPCN, 1989)

2.Lundgren : Wave Shock Pressures (Proc Symp Res on Wave Action, Delft, 1969, vol 2, paper $\left.n^{\circ} 4\right)$ 